Research Article

\title{
Cooperative Multi-Robot FrameworkUsing Artificial Immune System and Fuzzy Logic
}

\author{
Muhammad Saeed Shah ${ }^{1}$, Fazal Nasir ${ }^{2}$, Sana U1 Haq ${ }^{1}$ and Muhammad Tahir Khan ${ }^{2 *}$ \\ ${ }^{1}$ Department of Electronics, University of Peshawar, Pakistan; ${ }^{2}$ Department of Mechatronics Engineering, University of \\ Engineering and Technology Peshawar, Pakistan.
}

Abstract: The human immune system is a highly sophisticated system of biological structures that
defends the human body against harmful antigens. In this paper, the concept of biological immune
system, idiotypic network theory and clonal selection theory are applied in multi-robot cooperation.
Idiotypic network theory governs the stimulation and suppression of an antibody in response to an
antigen. Clonal selection theory represents how immune cells mutate and proliferate into memory
cells. The methodology presented in this paper is autonomous and de-centralized. In the proposed
system, the robots work effectively to complete a task either alone or co-operatively. Specifically,
the coordination and communication among various robots are established based on the concept of
immune system, idiotypic network theory and clonal selection theory. The developed methodology
is successfully implemented through simulation. The experiments show that the simulated robots
completed all tasks effectively.
Received: January 12,$2020 ;$ Accepted: May 23, 2020; Published: June 18, 2020
*Correspondence: Muhammad Tahir Khan, Department of Mechatronics Engineering, University of Engineering and Technology Peshawar,
Pakistan; Email: tahir@uetpeshawar.edu.pk
Citation: Shah, M.S., F. Nasir, S.U. Haq and M.T. Khan. 2020. Cooperative multi-robot framework using artificial immune system and fuzzy
logic. Journal of Engineering and Applied Sciences, 39(1): $01-13$.
DOI: http://dx.doi.org/10.17582/journal.jeas/39.1.01.13
Keywords: Multi-robot cooperation, Idiotypic network theory, Clonal selection theory, Artificial immune system

\section{Introduction}

W hile completing complex tasks in a dynamic environment, a single robot faces certain operational constraints. To improve the overall system performance and to reduce such limitations, the idea of multi-robot system (MRS) has been proposed (Khan et al., 2014). An MRS is composed of a set of self-organizing robots which seek to find an appropriate solution for a complex task in an autonomous manner. The robots in an MRS typically possess some degree of artificial intelligence and have local observations. They observe variations in the environment and in other robots, and take measures built on this knowledge.
Co-ordination and co-operation in an MRS may be described as "the group members accomplishing the tasks together in an organized manner to complete a global task either that cannot be completed by a single robot, or whose efficiency and performance can be improved by using more than one robot". An individual robot in such a system not only completes its own task, but also looks for and communicates with other group members to identify if there are more essential and crucial tasks that need to be completed in a timely manner.

Numerous algorithms and architectures have been developed and analyzed for multi-robot co-operation (MRC) which include market-based approaches, 
biology inspired methodologies, and behaviourbased architectures. The development of artificial immune system (AIS) is comparatively advanced and is progressing swiftly. The AIS concept has been incorporated in several intelligent systems including computer security (Forrest et al., 1994), fault diagnosis (Ishida, 1993), and MRSs (Khan and De Silva, 2009; Gao and Luo, 2008).

The idiotypic network theory proposed by Jerne suggests that an immune system has antibodies that not only recognize the foreign invaders (antigens) but can also recognize other antibodies (Jerne, 1974; Farmer et al., 1986). This leads to an extremely effective and efficient immune system that consists of a network of different antibodies.

The mutual interaction of robots in a multi-robot framework (MRF) for accomplishing various tasks and their individual task performing capabilities often suffer from the challenges of communication among the robots and underutilizing the robot's capabilities to achieve the optimal performance. This necessitates the need of a novel and more promising solution to this problem as compared to the existing approaches.

In this paper, the concepts of artificial immune system and fuzzy logic are utilized to develop a hybrid architecture for MRC. The idiotypic network theory and clonal selection theory are utilized to establish co-operation and coordination among a group of heterogeneous robots. Specifically, fuzzy logic is incorporated in the architecture to calculate the binding affinity and to select the best robot among the robotic team for the co-operative task accomplishment.

This paper is organized as follows. Section 2 presents a literature survey. The concept of immune system is introduced in Section 3. The proposed methodology for MRC is given in Section 4. Simulation results are discussed in Section 5, while the conclusion and future work are given in Section 6.

\section{Related work}

Research in the area of MRC is rapidly progressing and several algorithms and architectures have been developed. The existing techniques have both advantages and disadvantages. The progress made in the area of MRC is presented in this section.
A disseminated architecture for MRC scheme was proposed by Botelho and Alami (1999). The architecture for co-operative mechanism was based on contract net protocol. The allocation of tasks among different robots and their realization was discussed and a co-operative mechanism was established for dealing with future events that are possible but could not be predicted with certainty. Chaimowicz et al. (2001) developed a framework that was decentralized, and the robots always follow a single robot known as a leader. The robots coordinate with each other through communication. An additional feature was incorporated to change the leader robot during task performance. A software framework named as ALLIANCE for the MRC with the capabilities of robust control and fault tolerance was developed by Parker (1994). In that framework, the performance of robots in the team was improved through learning. A biology-inspired architecture for MRC was developed by Yingying et al. (2003). To solve the problems of optimization and distributed control for MRC, the proposed architecture applied the idea of the cooperation of ants in acquiring their prey. The main problem addressed in the developed architecture was the number of robots required for the accomplishment of a particular task, which was solved by applying the concept of stigmergy from ant societies. Sariel and Balch (2006) proposed a control framework for multi-robot transportation known as DEMiR-CF. The developed architecture was intended for the execution and accomplishment of any difficult task. The approach integrated a co-operative framework for multi-robot task allotment and response to incidents. Auction-based task allocation approach for a multirobot cooperative system was developed by Gerkey and Mataric (2002). The approach was based on mediation process which incorporated contract net protocol. The approach was successfully implemented in the lightly and strongly integrated scenarios of multi-robot cooperation. Kuniyoshi et al. (1994) proposed a vision based approach for the MRC. The proposed control framework, called as collaboration by observation, had been successfully employed in real robots having actuators and sensors without the central control and communication. A multi-robot cooperative mechanism that was motivated by the biological immune system was presented in by Khan and De Silva (2013). The architecture was based on Jerne's idiotypic network theory. The selection of most appropriate robot for a cooperative task was based on Formers mathematical model of idiotypic network 
theory. A biology-inspired distributed system for MRC was proposed by Kube and Bonabeau (2000). The behaviour of ants was examined while they were transporting food to their nests and was applied in multi-robot cooperative systems. Ants reposition themselves if a deadlock is created while transporting food. Similarly, in the proposed architecture the robots repositioned themselves while performing a box pushing task when a deadlock was created. In our previous work a control framework was developed for the MRC based on the concept of artificial immune system (Khan et al., 2014). The architecture was effectively employed in a set of heterogeneous robots for transportation.

\section{Immune system}

Biological immune system: Biological Immune System (BIS) is a combination of organs, cells and tissues that work jointly to defend the body from the attacks of harmful intruders known as pathogens. Human body offers a perfect environment for several pathogens like bacteria, parasites and viruses that can produce illnesses. It is the duty of immune system to avoid the pathogens or diminish them. It can identify and memorize millions of invading pathogens. It comprises cells that produce secretions to discover and wipe out virtually any kind of attacking pathogens.

The organs of an immune system are arranged all over the body. They are called lymph nodes. Lymph nodes home immune cells known as lymphocytes. The lymphocytes can be categorized as T-lymphocytes and B-lymphocytes. The lymphocytes work collectively and can affect the functions of each other. However, their functions are different in the immune system. $\mathrm{T}$-lymphocytes are further divided into $\mathrm{T}$ helper cell and $\mathrm{T}$ killer cell. $\mathrm{T}$ helper cells communicate with other immune cells and coordinate the immune response. Some of the T helper cells activate B cells to generate antibodies (Kelly, 2007). B-lymphocytes on the other hand generate and release antibodies into the blood. The antibodies recognize and eliminate the foreign antigens that enter the body. Antibodies consist of four chains: two heavy chains and two light chains. The heavy and light chains are pooled to obtain a Y-shaped formation, as given in Figure 1. Each heavy chain and light chain can be characterized by two regions, variable and constant. The variable region, also called paratrope, is composed of the upper part of the heavy and light chains. The paratrope is different for every antibody and can recognize the antigen. The constant region is composed of the remaining of heavy and light chains, which is responsible for defining a suitable mechanism for the destruction of antigen. An antigen penetrates the blood stream and stimulates certain immune cells (whose paratrope matches the antigen's epitope). The antibody's paratrope on the surface of the cells is paired to the antigen's epitope, and thus the antibody connects itself to antigen. The antibody alone destroys the antigen or with the assistance of another antibody while working cooperatively.

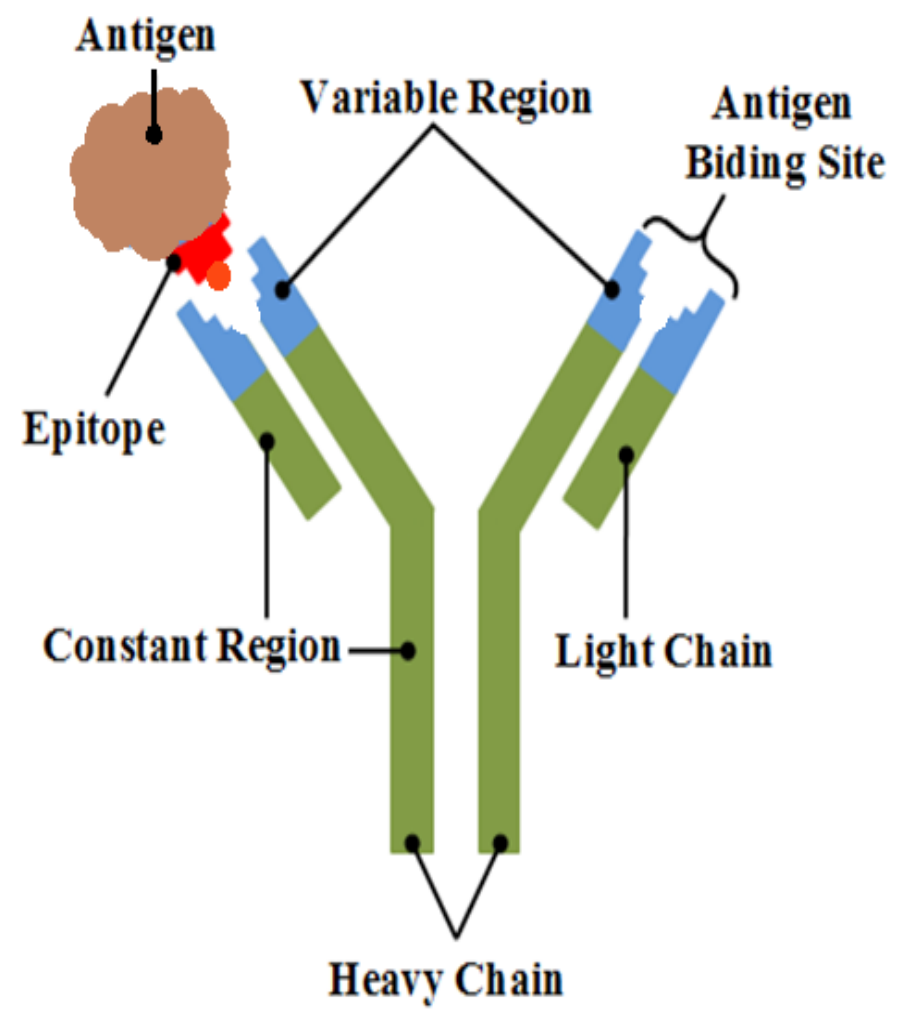

Figure 1: Antibody's structure.

The antibody's paratrope recognizes the epitope of an antigen with a specific binding affinity that depends on the matching potency amongst epitope and paratrope. A low affinity is generated if a paratrope of the antibody does not perfectly suit antigen's epitope. The perfect matching results in greater affinity, and hence the response of the immune system is more effective towards the antigen.

In addition to lymphocytes, an immune system stores plenty of phagocytes which capture and process the invading antigens. They attract the attention of matching lymphocytes by presenting the bits of the processed antigens to them.

Jerne's idiotypic network theory: The immune network theory proposed by Jerne (1974) states that 
an immune system can activate and recognize its own elements even if the antigens are absent. As per this theory, every antibody in immune besides paratrope has also idiotope on its surface that is recognizable for another antibody's paratrope, and thus can form a network of Figure 2.

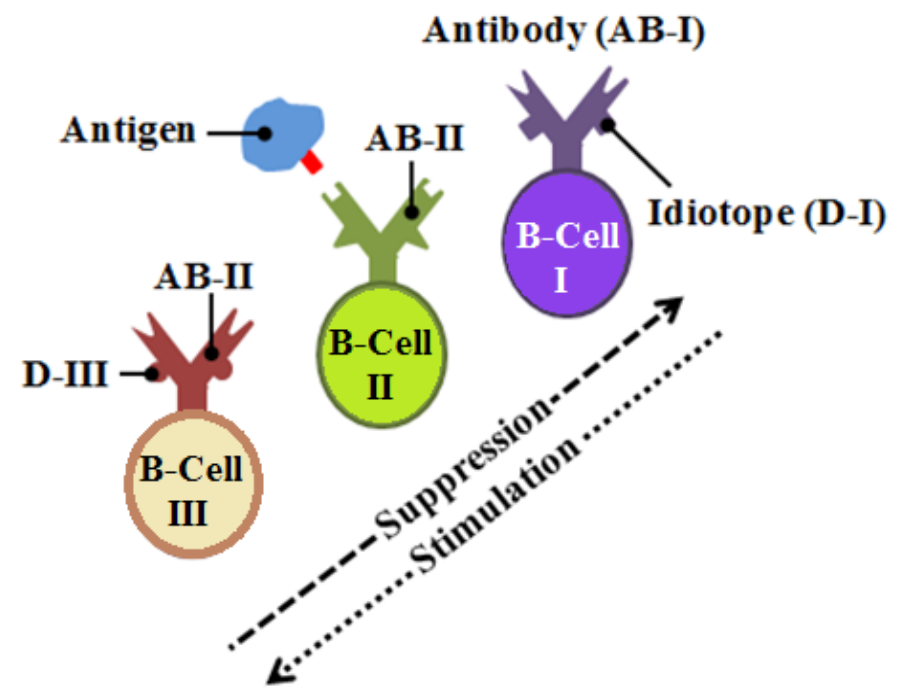

Figure 2: Idiotypic network theory.

The antibody AB-II on B-lymphocyte II matches the epitope of an antigen, and AB-II matches the idiotope D-I of an antibody AB-I on B-lymphocyte I. For this reason, the idiotope D-I is said to be internal image of the antigen. Thus antibody AB-II is capable of recognizing the AB-I and antigen. If the antibody's paratrope matches the idiotope of another antibody, it is triggered. On the contrary, when the idiotope of an antibody is known by the paratrope of another antibody, the antibody is inhibited. If a cell receptor identifies an antigen, it results in cell creation and system stimulation.

Bona (2018) discussed the idiotypic connection between the self and non-self antigens. In view of the extensive idiotype (ID) connectivity between self and non-self antigens, the deletion of such auto reactive clones would be impossible. The author proposed a model whereby autoimmunity was initiated by the normal operation of ID networks. It was observed that an anti-ID in one antigen system was potentially harmful autoantibody in another. Landmann et al. (2017) proposed a mathematical model for the idiotypic network that was self tolerating and autoimmune. A single node indicated B-lymphocytes clones and antibodies of the corresponding idiotype in the network and its encoding was done by a bit string in the network. The established links among the nodes were the representation of the clones that had idiotype with complementation. The survivability of a clone was dependent on the nodes in its neighborhood that must exist in moderate amount rather than in scarcity or in abundance. The network was powered by the flow of the lymphocytes that had idiotype generated in a random manner from the bone marrow.

\section{Clonal selection theory}

The successful identification of an antigen by a cell receptor causes the cell cloning, as shown in Figure 3. As a result of this replication, a cell proliferates (divides) and matures into plasma cells. This increases the concentration of antibodies that recognize and eliminate the antigens of a given type. The immune system is therefore able to eliminate the recurring antigens at higher efficiency and speed. This more rapidly response is termed as secondary reaction. In addition, the high affinity cells that are aroused by the antigen are chosen to become memory cells. In future responses, these memory cells play a pre-eminent role against similar antigens (De Castro and Zuben, 2000; Coutinho, 1989).

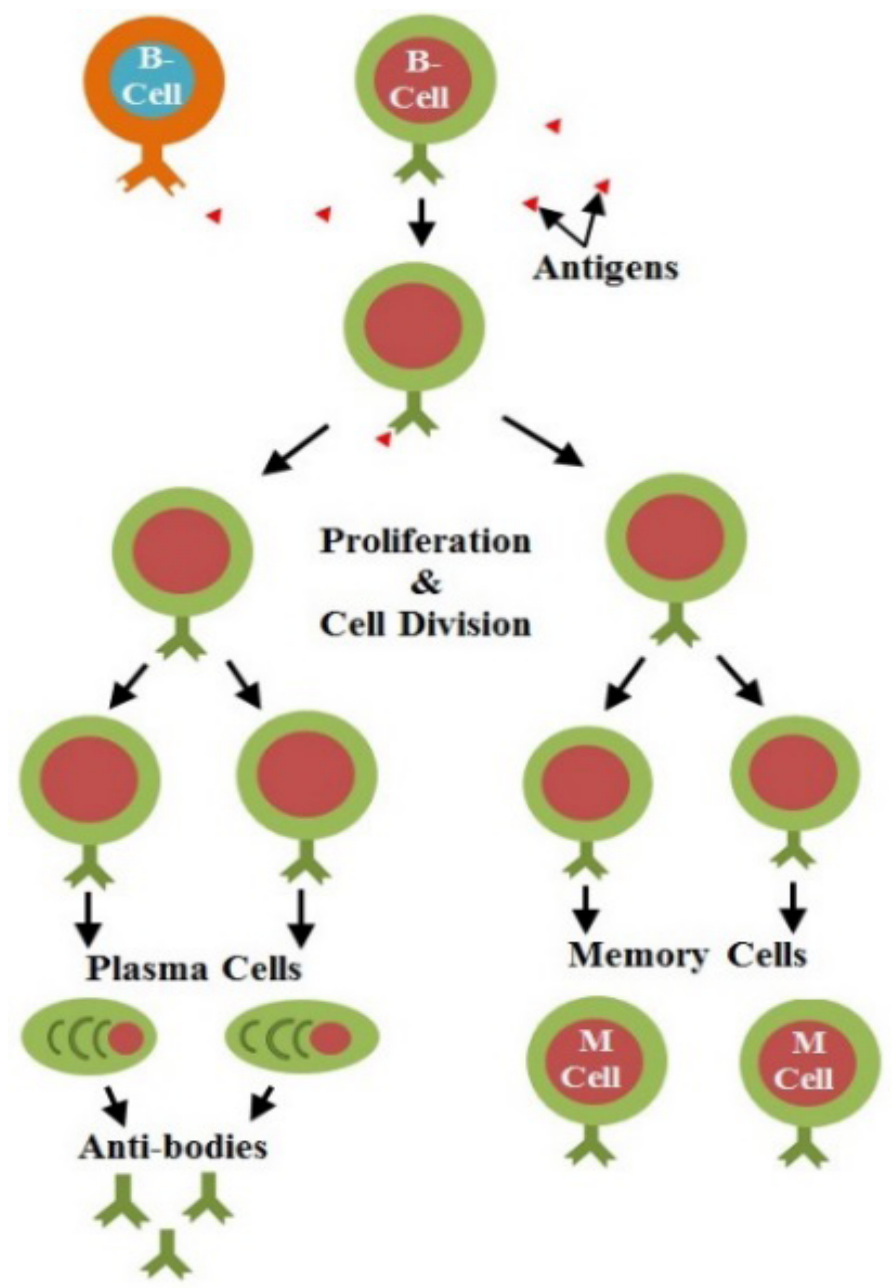

Figure 3: Clonal selection theory. 
Several features of the adaptive immune system, such as diversity adequacy, discriminating between self and non-self and maintaining the memory of immunology, are explained by Yang et al. (2019) using the clonal selection algorithm (CSA). The CSA provides proliferation and elimination of the cells that possess high affinitive. The CSA also helped in the identification of cells with low affinity by making use of the change in their receptors. For this reason, such cells are less subjected to the change and editing in their affinity, and therefore only the cells with high affinity are selected most of the times while cells with low affinity are discarded. The artificial immune system that is inspired by the biological immune system of the human being is a computation model in various forms. The CSA, being it's one of the forms, can be applied to solve a number of problems involving optimization. However, the complex optimization problems that involve CSA often have the issues of losing the diversity, unstable converging behaviour and the stagnation process (Zhang et al., 2019). These issues were overcome by proposing an improved version of the algorithm that utilized a diverse population and solved the problem of unstable converging behaviour. In addition, the searching ability was improved by introducing a mutation strategy that can be controlled adaptively.

\section{Materials and Mathods}

\section{Multi-robot cooperation problem}

The proposed framework is based on the concept of artificial immune system which mimics the function and structure of the human immune system to cope with the task accomplishment individually as well as collectively by the robots. The proposed framework for the MRC contains three kinds of robots: one type represents phagocytes known as $\mathrm{P}$-robots, another type represents $\mathrm{T}$ cells (helper) known as T-robots, while the third one represents $B$ cells known as B-robots. The purpose of P-robots is to search and scoute, and they are homogenous. Their job is to search for the duties in the environment and report it to B-robots. T-robots are also homogenous and are housed in charging stations (Lymph nodes). They are used to charge B-robots, thus ensuring uninterrupted operation of B-robots. B-robots performs both the task search and accomplishment, and they are heterogeneous.

Once a P-robot detects a task, it notifies the B-robots regarding the required capabilities and the location of the job, and then starts exploring the environment for another task. On the other hand if B-robot finds a task, it tries to accomplish it alone, given that it has the abilities to handle the task. However, if it is unable to accomplish the task alone, it will send a help signal to ask for the help of other B-robots. The robot that sends the help signal is called the initiating robot. The help signal comprises of data about the task location and the capabilities required to tackle the task. The B-robots in the search state will reply to the help call and will verify their abilities list to determine their suitability for the task. Those robots that can handle the task will determine their binding affinity. A robot in the team with highest binding affinity is chosen to help in accomplishing the task. The robot selected for the help is known as a helping robot which will co-ordinate with the initiating robot to work cooperatively.

If a B-robot finds a task that it cannot handle due to insufficient capabilities, it will inform other robots in the search state about the task and its capability requirement. If all the B-robots in the explore state are not capable of handling the task, then the B-robots in the busy state will be informed. If a B-robot in the busy state can handle the task, it will respond to the signal and will accomplish the task after completing the present task. If the B-robots in the busy state are also unable to handle this task, then the initiating robot will recognize it as a new task. The initiating robot will then generate a specific response. In the specific response, the initiating robot manipulates its capabilities with its teammates, and generates a specific capability that can handle a complex task. The helping robot in the specific response is selected based on the binding affinity function. The robots after completing a task will save information about the task in their memory. They will also send a message to all the remaining B-robots to save information about this task. Next time when the same complex task is found, the B-robot will check its memory and will directly generate a specific response instead of searching for B-robots in the system. As a result, the task will be completed more efficiently and quickly. Figure 4 shows the complete framework of multirobot cooperation based on AIS.

While performing a task the batteries of robots will deplete. If the battery of a P-robot or a B-robot depletes below a specific threshold, it will go to the 
charging base to restore its battery. However, if the battery of a B-robot depletes below the threshold while it is performing a task, it will send a signal to the $\mathrm{T}$-robot for charging its battery. $\mathrm{T}$-robot that has a mobile charger will reach the B-robot and will charge up the battery. The framework for charging the battery of a robot is shown in Figure 5.

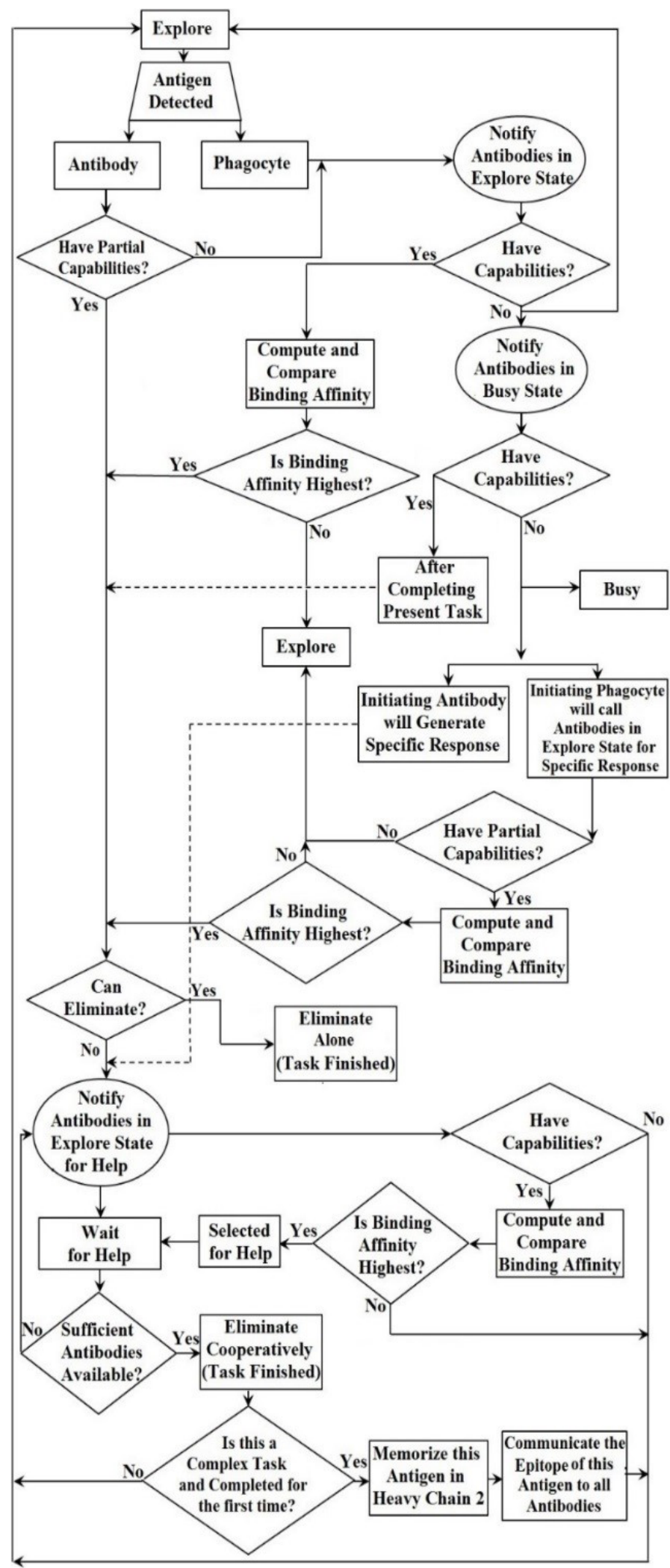

Figure 4: Control framework for $M R C$.

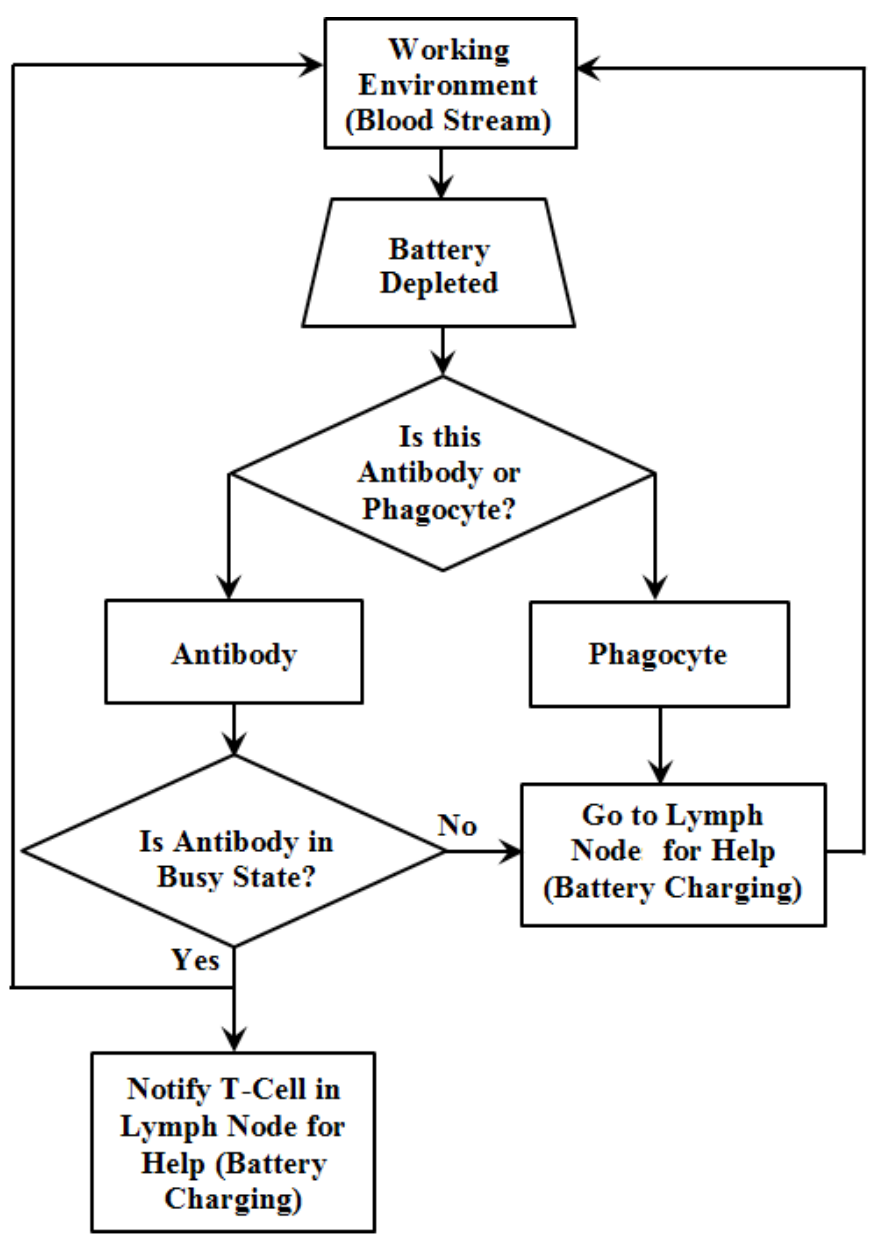

Figure 5: Flowchart for battery charging of a robot.

\section{Biological immune system and multi-robot cooperation analogy}

The capacities of B-robot are equivalent to the antibody's paratrope. The tasks in the environment are like antigens, and aspects of the task are equivalent to the antigen's epitope. In the case of multi-robot system, the robots work in an unknown and dynamic environment that contains both dynamic and static obstacles. These obstacles are like self-antigens. In an immune system, the immune cell only destroys the non-self-antigens while leaving behind the selfantigens. Analogously, in multi-robot cooperation, the robots avoid each other and obstacles, and seek to complete the tasks present in the environment.

\section{Phagocytes and P-robots}

Phagocytes in an immune system take and process the antigens. They portray the epitopes of the treated antigens in such a way to attract the interest of the identical B cells. Similar to phagocytes, P-robots in multi-robot co-operation have a vision system. They explore the location for tasks and obtain information about them, e.g., the location of the task and its capability requirements. They share this information 
with other B-robots (antibodies).

$T$ (Helper) cells and $T$-robots

$\mathrm{T}$ cells in an immune system communicate with other immune cells and co-ordinate the immune response. Some of these cells activate B cells to generate antibodies. Analogous to T (helper) cells, T-robots in multi-robot cooperation have a mobile charger and they charge the batteries of B-robots whenever it is required.

\section{$B$ cells and B-Robots}

In an immune system, a $B$ cell squirts unique $Y$ shaped structures called antibodies. The antibody contains both the light and heavy chains. A B-robot, similar to an antibody, has a set of light chains (LC1 and LC2) and a set of heavy chains ( $\mathrm{HC} 1$ and $\mathrm{HC} 2$ ), as shown in Figure 6. The LC1 represents the form of robot at an instance. A robot may be in search or in working mode at a specific time. The light chain LC2 contains the general sensory data that includes the speed of robot, the orientation of robot relative to the task, the frequency of task accomplishment and the obstacles in front of robot. The heavy chain HC1 stores the skills of a robot including its software capacities, e.g., the vision system, and hardware capabilities, e.g., arms, wheels, bumpers and gripper. The heavy chain HC2 is reserved for a specific response and it represents the capability requirement for a complex task that is accomplished through a specific response.

\section{Lymph nodes and charging stations}

Lymph nodes in an immune system resides lymphocytes. Similar to lymph nodes, charging bases are used in this research to charge the batteries of robots. In addition, T-robots are also housed in the charging stations.

\section{Binding affinity function}

Binding affinity in the context of immune system is the strength with which the antigen's epitope matches the antibody's paratrope. In MRC, the concept of binding affinity is utilized for the selection of most skilled and appropriate robot to accomplish a given task. It is a function of gap between a robot and a certain task $\left(D_{r t}\right)$, the velocity of the robot $\left(U_{r}\right)$, the charge in the battery of a robot $\left(B_{r}\right)$, obstacles between the robot and the task $\left(H_{r t}\right)$, and the performance rate of the robot $\left(P_{r}\right)$.

$$
B A=f\left(D_{r t}, U_{r}, B_{r}, H_{r t}, P_{r}\right) \quad \ldots(1)
$$

\section{\begin{tabular}{l|l} 
LC1 & LC2
\end{tabular} \\ HC1 \\ HC2 \\ Mode Sensory Data Capabilities \\ Capabilities \\ Requirement of \\ Complex Tasks}

Figure 6: Antibody's (B-robot) light and heavy chains.

In this research work, Manhattan distance is used to determine the distance between a motivated robot and the job. Manhattan distance is resilient to noisy data and is more efficient in terms of computation.

Manhattan distance is defined by.

$$
D_{r t}=\left|x_{r}-x_{t}\right|+\left|y_{r}-y_{t}\right| \ldots
$$

Binding affinity is also dependent on the robot's performance rate. If a task is successfully accomplished by a robot, its performance rate is enhanced.

\section{Fuzzy logic-based approach}

An unknown environment cannot be predicted accurately and thus cannot be modelled. The Fuzzy logic is very appropriate for such kind of environments because it does not require any modelling. A block diagram for fuzzy binding affinity is shown in Figure 7. The key steps in calculating the binding affinity through fuzzy logic are fuzzification of the input variables, evaluation of the rules and defuzzification of the fuzzy output variable (Karray and De Silva, 2004; Dubois and Prade, 1980).

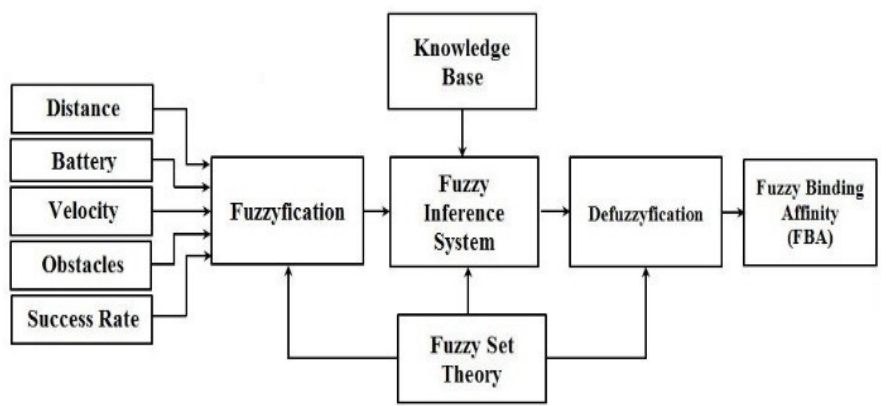

Figure 7: Block diagram of the proposed algorithm for fuzzy binding affinity.

The proposed algorithm takes the distance between the robot and the task $\left(D_{r}\right)$, the velocity of $\operatorname{robot}\left(U_{r}\right)$, the charge in the battery of a robot $(B)$, obstacles between the robot and task $\left(H_{r r}\right)$ and the success rate of robot $\left(P_{r}\right)$ as input variables. These inputs are then fuzzified in the fuzzy plane. Figures 8 and 9 show the associated membership functions with the input and output fuzzy sets, respectively.

The triangular membership functions were used 
because they are computationally cheap as compared to other complex functions. In addition, it is observed that the results are not affected significantly by using the triangular membership functions as compared to complex functions.

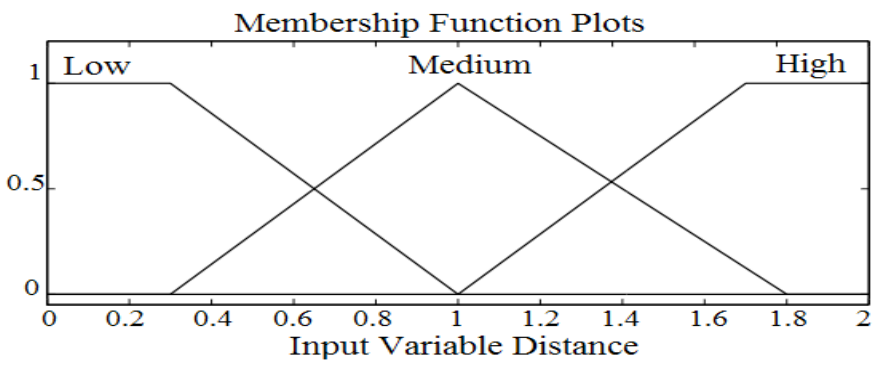

(a)
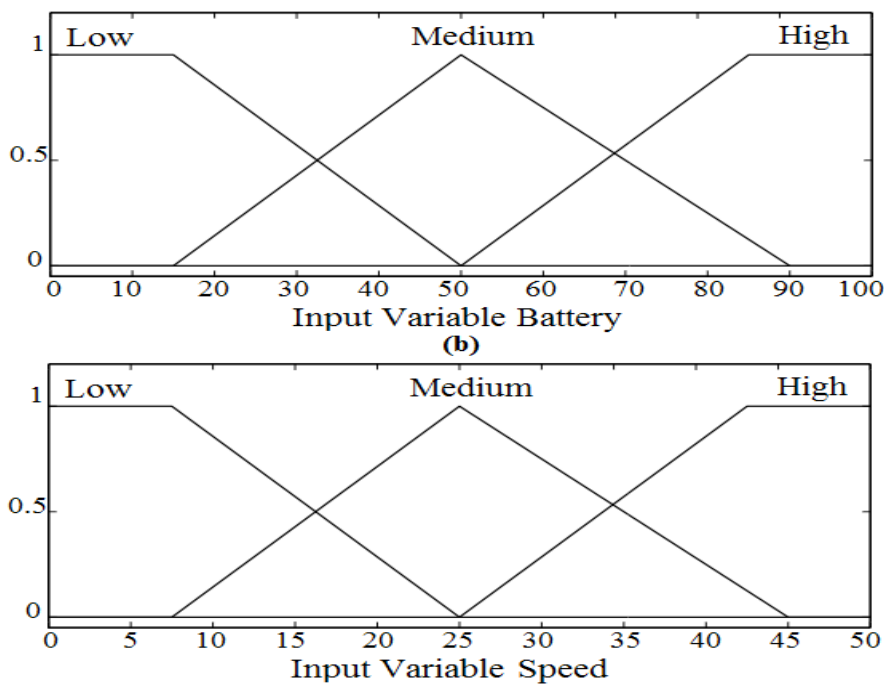

(c)

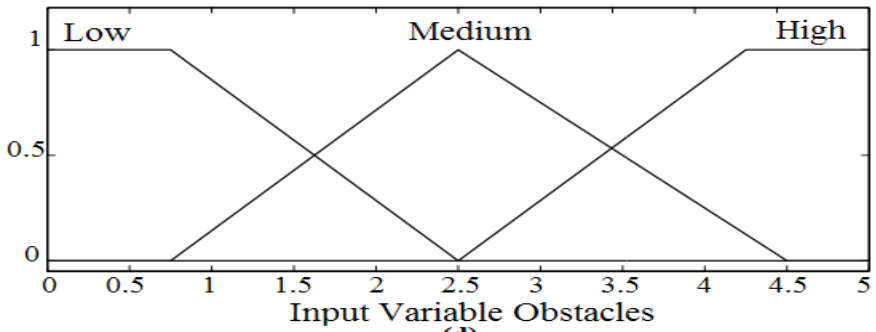

(d)

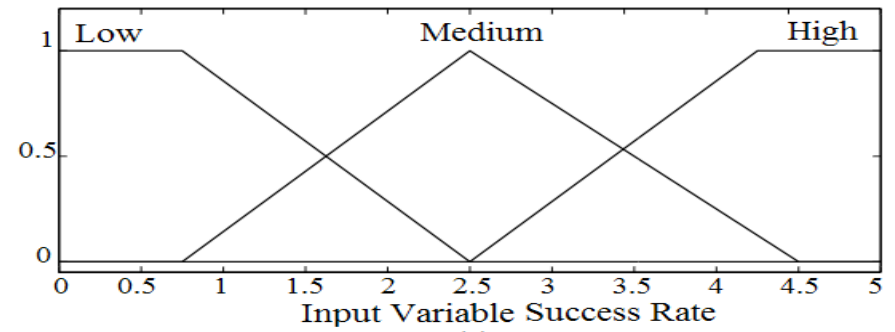

(e)

Figure 8: Input membership functions:(a) Distance, (b) Battery, (c) Speed, (d) Obstacles, (e) Success rate.

A knowledge base containing a set of 243 rules evaluates the fuzzified inputs to measure the binding affinity in the fuzzy plane. A sample of the rule base is:

If the Distance is 'Low' and the Battery is 'High' and the Velocity is 'High' and the Obstacles is 'Low' and the Success Rate is 'High' then the Binding Affinity is 'High'.

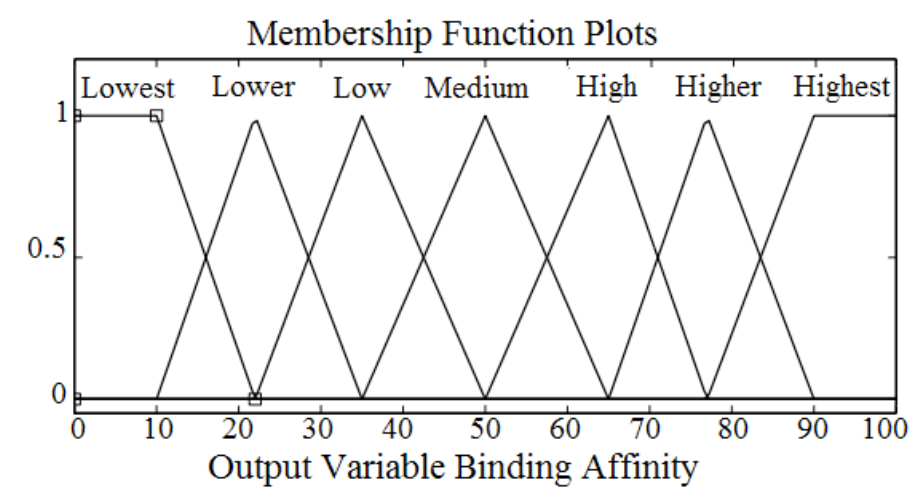

Figure 9: Output Binding Affinity membership function.

The output of the fuzzy inference system is in the fuzzy plane. The defuzzification of the output variable is performed using the centroid method to obtain a crisp value of the binding affinity.

\section{Network theory and multi-robot cooperation}

Non-specific response: In non-specific response, upon identification of a topic, a B-robot tends for its accomplishment. If the robot is unable to execute the task, it will contact other B-robots and will cooperate with them to complete the task. In the first step, the B-robot that initiates the task completion matches its capabilities with those of the robot whose capabilities are known to some extent. Figure 10 depicts the matching of paratrope of the antibody (robots capabilities) with the task capabilities of the antigen.

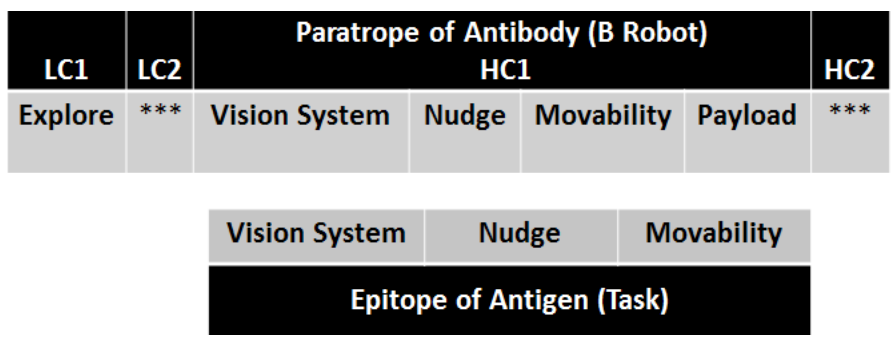

Figure 10: Comparison of the paratrope of antibody (B-robot) with epitope of task.

The comparison of the task capability requirements (epitope) and the robot's capabilities (paratrope) is accomplished in all of the alignment. If the robot capabilities and the task capabilities match, the robot is stimulated and will proceed to complete the task.

The initiating robot transmits a help signal to B-robots that act as antibodies if it is unable to complete the task alone, which comprises of information related to June 2020 | Volume 39 | Issue 1 | Page 8 
the task i.e., the location of the antigen and its epitope that appears to the robots in the idiotope form. In order to respond to the help, signal, the neighbour B-robots that are in the explore state are activated and their paratrope match with that of the robot that initiates the initiative as shown in Figure 11.

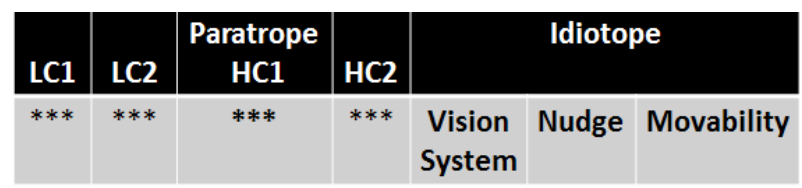

\begin{tabular}{|c|c|c|c|c|c|c|}
\hline LC1 & LC2 & Parat & rope of & $\begin{array}{l}\text { Antibody (B } \\
\text { HC1 }\end{array}$ & bot) & HC2 \\
\hline$* * *$ & $* * *$ & $\begin{array}{l}\text { Vision } \\
\text { System }\end{array}$ & Nudge & Movability & Payload & $* * *$ \\
\hline
\end{tabular}

Figure 11: Matching of the antibody (B-robot) paratrope and idiotope.

B-robots that have all the completely capable take part in the simulation process and the rest go into the explore state are stimulated while the remaining B-robots will go for exploration and searching the environment for another task.

Stimulation of the antibodies in an immune system happens when they come in direct contact with another antibody. However, in the present work of multirobot cooperation, the robots stimulate each other by mutual communication without physical contact. The binding affinity function is used to choose the most suitable robot during simulation. Every robot finds its binding affinity based on the data already captured and the robot that has the highest value of the affinity function is chosen for help. The helping and initiating robots mutually then communicate to perform the task.

If a P-robot finds a task, it messages the B-robots related to the position and the capability needed for the completion of the task. All the B-robots compute their binding affinity and the robot with the highest value of this function accomplishes the task.

Specific response: To generate a specific response, different B-robots (antibodies) manipulate their capabilities to build a specific capability that can handle a complex task. For example, if a robot locates a task that requires both nudge and picks capabilities but there is no robot in the system that can both pick and push the object. Then two robots, one having the capability of nudge will combine with a robot having the capability of pick and will generate a specific response for object transportation. At first, when a B-robot locates the complex task that it cannot handle, it will communicate it to other B-robots. If no robot in the system has the required capabilities for task completion, a specific response is generated by the initiating robot. In the specific response, the robot makes two portions of the capability requirements of the task. The first portion contains the capabilities that the robot can handle, while the second portion contains the capabilities that the initiating robot needs for task accomplishment. The initiating robot matches its capabilities (paratrope) with the first portion of the capability requirement and is stimulated. For the second portion of the capability requirement, the robot seeks help and transmits a help message to other B-robots. The help signal communicates the idiotope of the initiating robot to other B-robots. The idiotope in the specific response contains the second portion of the capability requirements of the task that the initiating robot cannot handle, as shown in Figure 12.

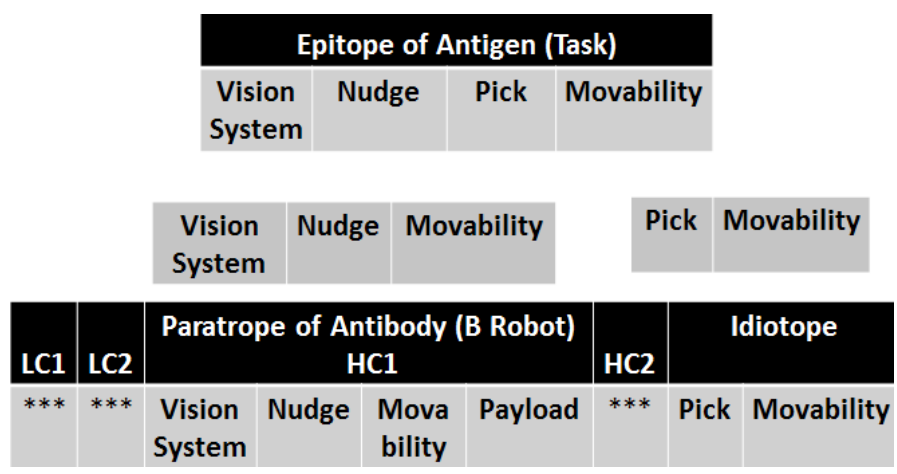

Figure 12: Response of the antibody to a complex antigen (task).

Those B-robots in the exploration mode and in the neighbourhood perform a capabilities comparison with the idiotope of the robots that initiated at first. The robots that have the essential capabilities are activated. The stimulated robots calculate their binding affinities and compare with each other. The best among them, which has the greatest binding affinity performs the helping process. The helping robot co-operates with the initiating robot to generate a specific response and complete the task.

Cloning: The clonal selection theory states that some of the B cells based on the total stimulation are selected and copies (cloning) of each selected B cell are created. In the present study, analogous to cloning, when B-robots complete a complex task through specific response, they memorize the capabilities of the task in the heavy chain $\mathrm{HC} 2$, as depicted in 
Figure 13. They also communicate the capabilities of this task to other B-robots in the system, and the remaining robots also memorize the capabilities of this task in their HC2. Next time when this task is detected, the B-robot will match the capabilities of this task in its $\mathrm{HC} 2$ rather than checking the entire system for the required capabilities. In this manner, the robot will directly stimulate a quick secondary (specific) response to complete the task.

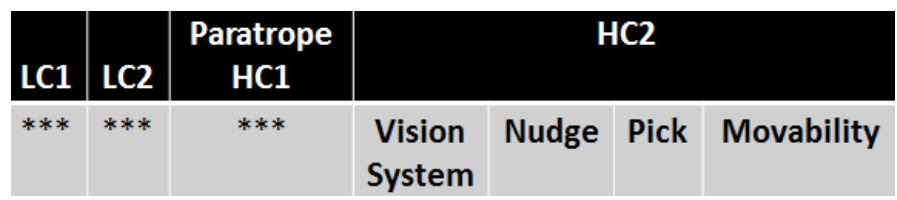

Figure 13: Heavy chain HC2 showing capabilities of the task accomplished through a specific response.

\section{Results and Discussion}

A simulation study was conducted to show the effectiveness of the developed methodology. The Java programming was used for simulation. A number of robots with heterogeneous properties are deployed to perform the simulation. The robots were assigned tasks of transporting number of objects with different complexity levels to a pre-defined goal location, as shown in Figure 14. The simulation considered three forms of robots (P-robots, B-robots and T-robots), five different objects to be moved, static obstacles, and three charging bases. P-robots and T-robots cannot transport objects, but they were used to facilitate the B-robots. P-robots explore the environment for objects and upon detection of an object they send information to B-robots. T-robots were used to charge the depleted batteries of B-robots, thus ensuring uninterrupted operation of the B-robots. Two types of B-robots were used in this research: R-type and G-type. The R-type robots can transport only R-type objects, while the G-type robots can transport only G-type objects. In addition, different types of B-robots can manipulate their capabilities to generate another capability and transport a complex object as well. The robots are capable of avoiding the stationary and moving obstacles during task performance.

In Figure 15, the time consumed by the robots and the effect of $\mathrm{P}$-robots and $\mathrm{T}$-robots are given. The average time taken to transport the objects when both $\mathrm{P}$-robots and T-robots are used is much smaller than when only each ( $\mathrm{P}$ or $\mathrm{T}$ ) robots or none of them are used. It is quite justified because with more resources, higher performance can be achieved. It is observed that each round of simulation the time for the robots to perform tasks varies and is not constant. This is because the robots search for the object as their working environment is unfamiliar and dynamic. In addition, it is likely that robots suffer from stationary and movable obstacles during their task performance that ultimately cause overshooting.

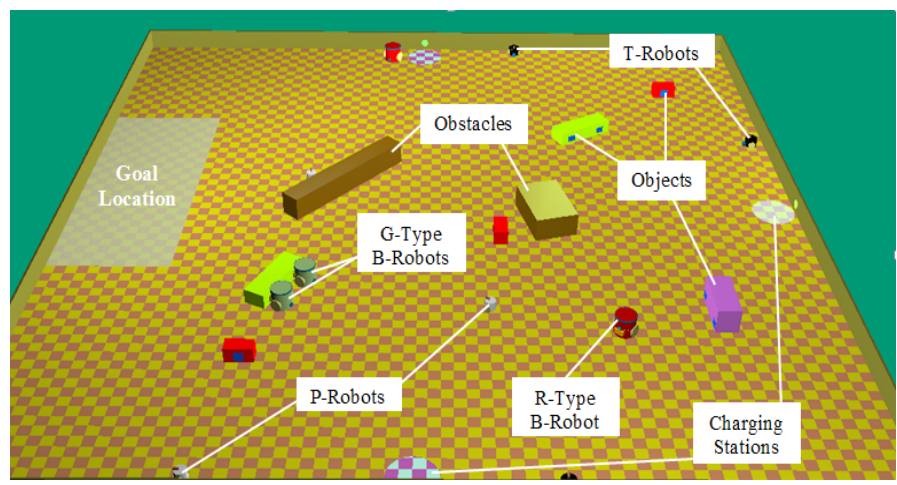

Figure 14: Simulation platform.

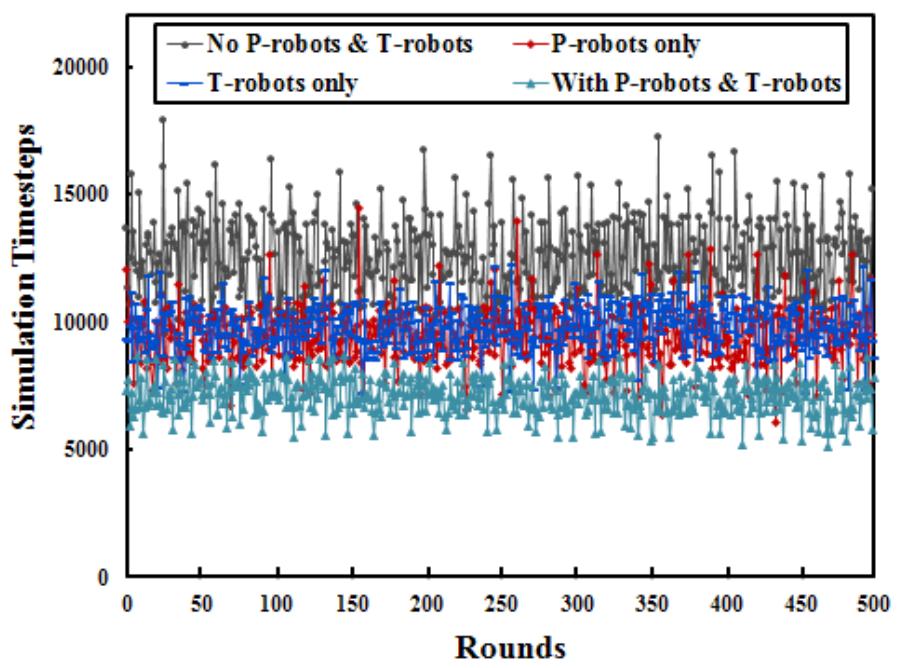

Figure 15: Time (stpes) spent in each run to complete the tasks.

Figure 16 shows the amount of total messages exchanged by the robots. In addition, the effect of $\mathrm{P}$ and T-robots is also investigated. It is observed that when only B-robots are used, the number of messages exchanged is lesser than when both $\mathrm{P}$ and $\mathrm{T}$-robots are used. This is due to the fact that without $\mathrm{P}$ and T-robots, it is highly likely that detection and transportation of an object by a special B-robot will be accomplished without communicating with other robots. If its battery is depleted during transportation, it will proceed to the charging station for charging and then the task performance will be followed. On the other hand, if a P-robot notices an object it will send the information to the B-robots, and in order to find a specialized and most suitable robot, many communication messages will be exchanged. If T-robots (charging robots) are used, then the 
communication burden will be further increased. This is because during transportation if the battery of a B-robot depletes, it will send a message to a nearby T-robot to charge its battery. As the location of the B-robot continuously changes during transportation, it continuously conveys its location data to the T-robot, and as a result many communication messages are exchanged.

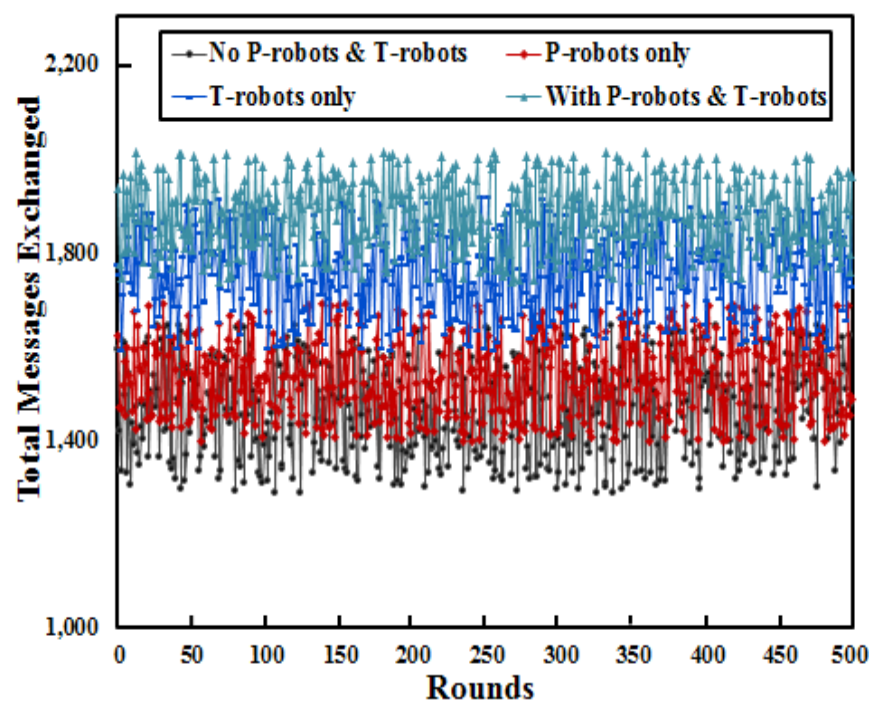

Figure 16: Total messages exchanged during task accomplishment in each run.

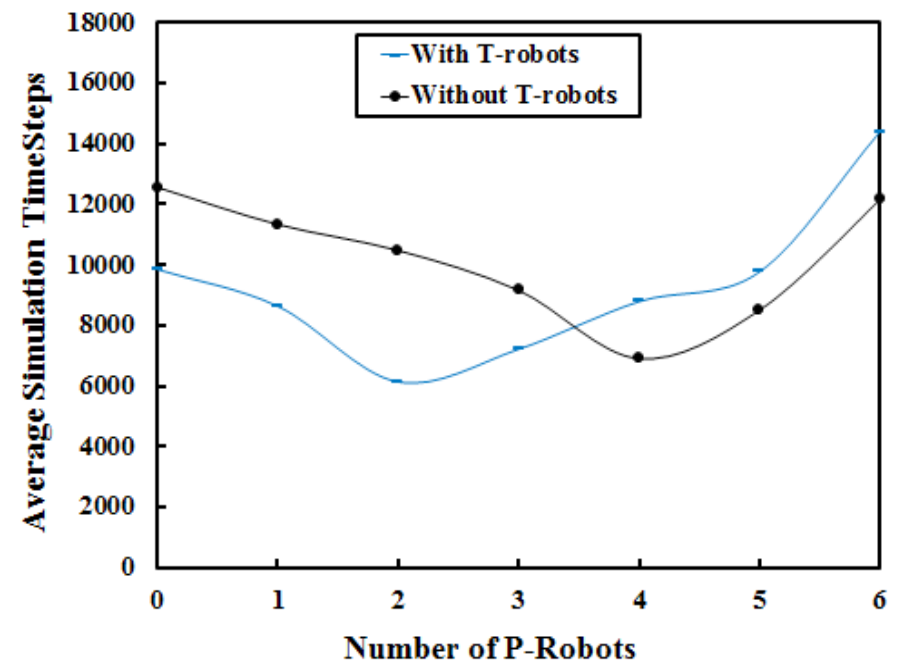

Figure 17: Average time (steps) taken to complete the tasks as a function P-robots.

Figure 17 shows the total time (steps) that robots take for task performance present in the environment as a function of P-robots. It is noted that as we improve the quantity of P-robots, the system efficiency with respect to time initially increases and then declines. The rise in the system performance is understandable since high performance is achieved with more resources. However, reduction in system performance may be related to obstacles among the robots themselves. Therefore, as the quantity of P-robots rises, the hurdles among robots increase the causes of delay. It is also clear that when no P-robot is there, more steps are involved which involves more time.

Figure 18 shows the total count of exchanged messages during the accomplishment of the tasks as a function of P-robots. It is observed that the communication messages rise with the surge in the quantity of P-robots. This is because with more $\mathrm{P}$-robots it is highly likely that a task will be detected by a P-robot. However, P-robots cannot perform tasks and they can only inform B-robots about the detected task. While choosing the best B-robot (to perform the task detected by a $\mathrm{P}$-robot), large number of messages are exchanged that contribute to the communication burden. However, by increasing the number of $\mathrm{P}$-robots up to a certain limit (three robots in the present case), the communication messages remain constant. This is because the tasks are always identified by $\mathrm{P}$-robots if the robots count increases from this particular value (three).

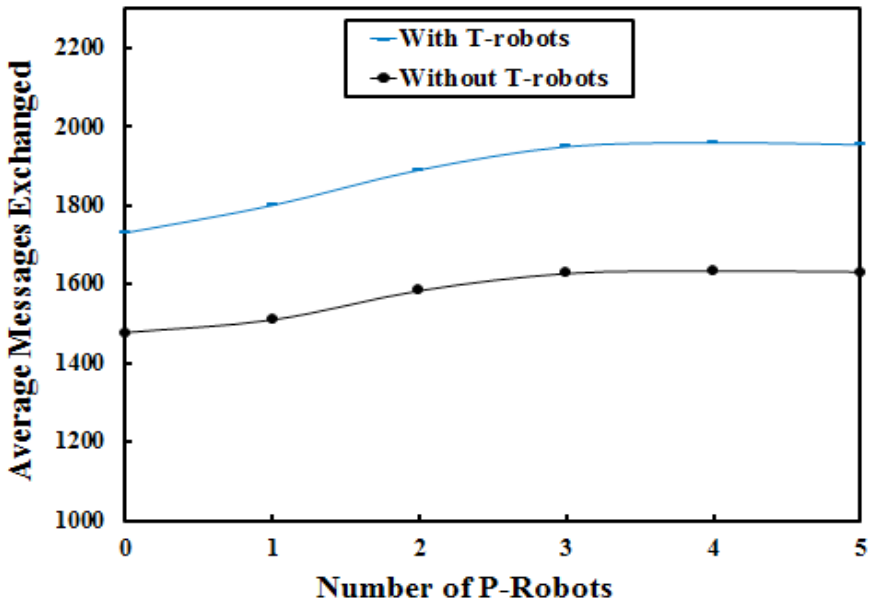

Figure 18: Average messages exchanged during tasks completion as a function of $P$-robots.

Figure 19 shows the step count taken for the sake of completing tasks as function of T-robots (charging robots). It is clear that increasing the T-robots count reduces the time required for the tasks completion. This is understandable because with more T-robots, a B-robot during transportation of a task can charge its battery with the help of T-robot (mobile charger). However, in case the count of T-robots is enhanced beyond a certain limit (three in the present case), the time required in completion of such tasks remains constant. This is because for this specific value (three), enough T-robots are available to charge the depleted batteries of all B-robots in the busy state. 


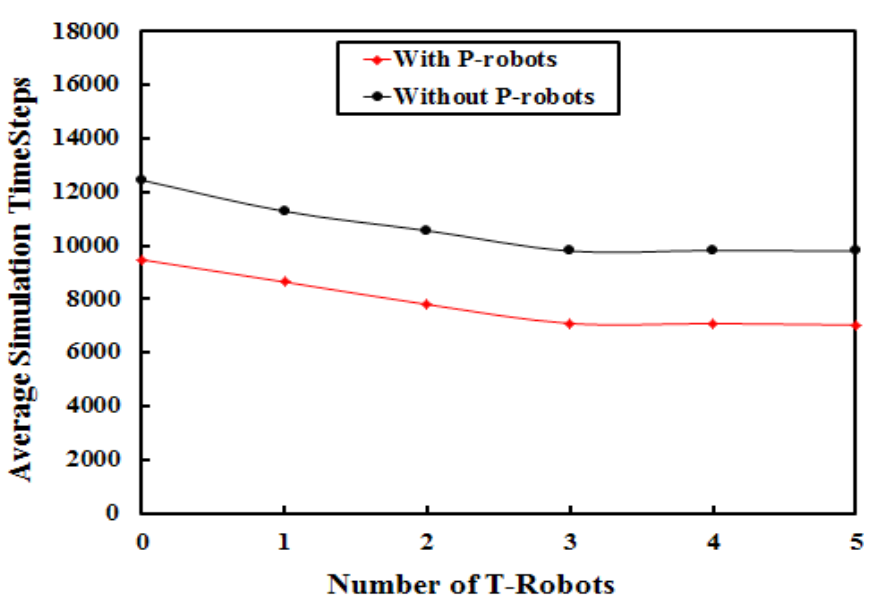

Figure 19: Average time (steps) taken to complete the tasks as a function T-robots.

Figure 20 shows the impact of powering the T-robots on the burden of communication. It is observed that when the number of T-robots is increased, the communication messages also increased. This is because if the battery of a B-robot depletes during transportation it will send a message to a nearby T-robot to charge its battery and will continue its job. As the location of B-robot continuously changes during transportation, it continuously sends its location to the T-robot and as a result many communication messages are exchanged.

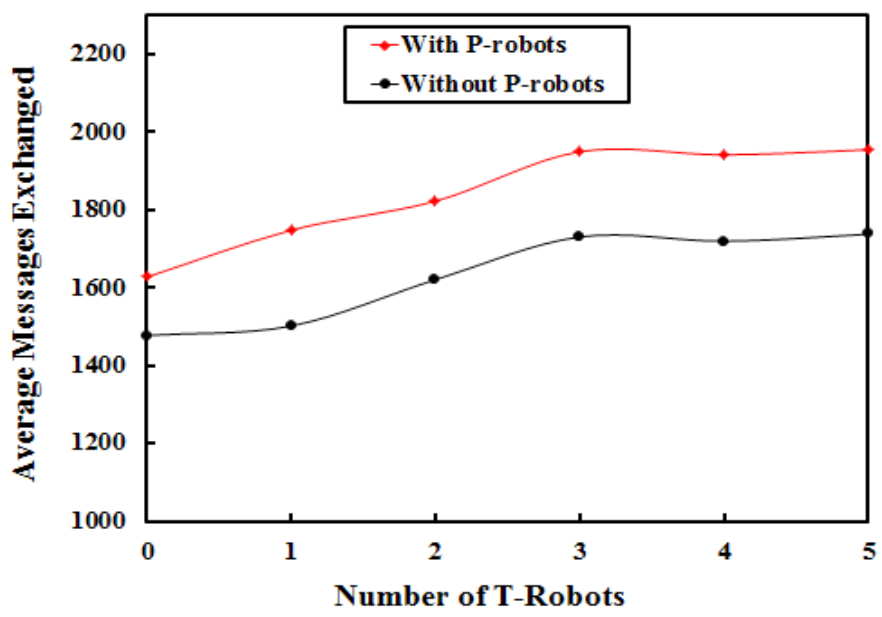

Figure 20: Average messages exchanged during tasks completion as a function of T-robots.

\section{Conclusions and Recommendations}

In this paper, MRC framework based on AIS and fuzzy logic was developed. The proposed architecture for MRC was established using the concept of general immune system and clonal selection algorithm. In addition, fuzzy logic was incorporated in the proposed architecture to select the most suitable robot among the team for a specific task accomplishment. The developed architecture is completely de-centralized and self-organized. To verify the effectiveness and feasibility of the developed control framework, a simulation study was conducted. Robots in the simulation study were assigned different tasks which constituted objects that had to be transported to an already defined locations. The simulation results revealed that the developed framework was effective and efficient in completing the given tasks in an unfamiliar and dynamic environment that contained both static and moving obstacles. The developed control framework can be conveniently implemented in physical robots working in a real physical environment.

Since the robots are powered by batteries that drain quickly, future investigation may add wireless charging of the batteries of these robots using energy harvesting techniques so that the network can be operated persistently. In addition, those tasks that robots cannot perform should be added intelligently to the system memory and a mechanism for providing solutions to such tasks will enhance the efficiency of the network to a next higher level.

\section{Novelty Statement}

In this research, the concept of biological immune system, idiotypic network theory and clonal selection theory are applied in multi-robot cooperation.

\section{Author's Contribution}

Muhammad Saeed Shah: Literature review, methodology, simulation, manuscript writing.

Fazal Nasir: Literature review, results analysis, manuscript writing.

Sana U1 Haq: Methodology, results analysis and interpretation.

Muhammad Tahir Khan: Concept, manuscript review

\section{Conflict of interest}

The authors have declared no conflict of interest.

\section{References}

Bona, C.A., 2018. Biological application of antiidiotypes. CRC Press, Boca Raton, USA. https://doi.org/10.1201/9781351070225

Botelho, S.C. and R. Alami. 1999. M+: A scheme for multi-robot cooperation through negotiat- 
ed task allocation and achievement. Proc. IEEE Int. Conf. Robot. Autom., Toulouse, France, pp. 1234-1239.

Chaimowicz, L., T. Sugar and V. Kumar. 2001. An architecture for tightly coupled multi-robot cooperation. Proc. IEEE Int. Conf. Robot. Autom., Seoul, South Korea, pp. 2992-2997.

Coutinho, A., 1989. Beyond clonal selection and network. Immunol. Rev., 110(1): 63-67.https:// doi.org/10.1111/j.1600-065X.1989.tb00027.x

De Castro, L.N. and F.J. Zuben. 2000. The clonal selection algorithm with engineering applications. Proc. GECCO Workshop Artif. Immun. Syst. Their Appl., Las Vagas, USA, pp. 36-37.

Dubois, D. and H. Prade. 1980. Fuzzy sets and systems. Aced. Press, Orlando, Florida.

Farmer,J.D., N.H. Packard and A.S.Perelson. 1986. The immune system adaptation and machine learning. Phys. D Nonlinear Phenomena, 22(13): 187-204. https://doi.org/10.1016/01672789(86)90240-X

Forrest, S., A.S. Perelson, L. Allen and R. Cherukuri. 1994. Self-nonself discrimination in a computer. Proc. IEEE Comput. Soc. Symp. Res. Secur. Privacy, Oakland, CA, USA, pp. 202-212.

Gao, Y. and Z. Luo. 2008. Dynamic task allocation method based on immune system for cooperative robots. Proc. World Cong. Intell. Contr. Autom. Chongqing, China, pp. 1015-1020.

Gerkey, B.P. and M.J. Mataric. 2002. Sold: Auction methods for multirobot coordination. IEEE Trans. Robot. Autom., 18(5): 758 - 768. https:// doi.org/10.1109/TRA.2002.803462

Ishida, Y., 1993. An immune network model and its applications to process diagnosis. Syst. Comput. Japan, 24(6): 38-45. https://doi.org/10.1002/ scj. 4690240604

Jerne, N.K., 1974. Towards a network theory of the immune system. Cell, 125: 373-389.

Karray, O. and C.W. De Silva. 2004. Soft computing and intelligent systems design: Theory, tools and applications. Pearson Addison Wesely, Essex, England.

Kelly, J., 2007. Understanding the immune system how it works. US Department of Health and Human Services, United States.

Khan, M.T. and C.W. De Silva. 2009. Immune system-inspired dynamic multi-robot coordination. Proc. ASME/IEEE Int. Conf. Mechatron. Embed. Syst. Appl. San Diego, CA, pp. 37-43.

Khan, M.T. and C.W. De Silva. 2013. Multi-robot cooperation using an immune system model for multi-object transporataion. Int. J. Robot. Autom., 28(1): 42-56. https://doi.org/10.2316/ Journal.206.2013.1.206-3649

Khan, M.T., Izhar, F. Nasir, U. Qadir and J. Iqbal. 2014. Artificial immune system based framework for multi-robot cooperation. Proc. Int. Conf. Comput. Sci. Educ. Vancour, Canada, pp. 50-55. https://doi.org/10.1109/ ICCSE.2014.6926429

Kube, C.R. and E. Bonabeau. 2000. Cooperative transport by ants and robots. Robot. Auton. Syst., 30(1): 85-101. https://doi.org/10.1016/ S0921-8890(99)00066-4

Kuniyoshi, Y., J. Riekki, M. Ishii, S. Rougeaux, N. Kita, S. Sakane and M. Kakikura. 1994. Vision-based behaviors for multi-robot cooperation. Proc. IEEE/RSJ/GI Int. Conf. Intell. Robots Syst., Munich, Germany, pp. 925-932.

Landmann, S., N. Preuss and U. Behn. 2017. Self-tolerance and autoimmunity in a minimal model of the idiotypic network. J. Theor. Biol., 426(3): 17-39. https://doi.org/10.1016/j. jtbi.2017.05.004

Parker, L.E., 1994. Alliance: An architecture for fault tolerant, cooperative control of heterogeneous mobile robots. Proc. IEEE/RSJ Int. Conf. Intell. Robots Syst., Munich, Germany, pp. 776-783.

Sariel, S. and T. Balch. 2006. A distributed multi-robot cooperation framework for real time task achievement. In: Distributed autonomous robotic systems. Gini, M. and R. Voyles (Ed.), Springer, Tokyo, pp. 187-196. https:// doi.org/10.1007/4-431-35881-1_19

Yang, Y., H. Dai, S. Gao, Y. Wang, D. Jia and Z. Tang. 2019. Complete receptor editing operation based on quantum clonal selection algorithm for optimization problems. Inst. Electr. Eng. Japan, 14(3): 411-421. https://doi. org/10.1002/tee.22822

Yingying, D., H. Yan and J. Jingping. 2003. Multi-robot cooperation method based on the ant algorithm. Proc. IEEE Swarm Intell. Symp., Indianapolis, IN, USA, pp. 14-18.

Zhang, W., K. Gao, W. Zhang, X. Wang, Q. Zhang and H. Wang. 2019. A hybrid clonal selection algorithm with modified combinatorial recombination and success-history based adaptive mutation for numerical optimization. Appl. Intell., 49(2): 819-836. https://doi.org/10.1007/ s10489-018-1291-2 